\title{
Response to select comments on the proposed paradigm shifts in running
}

\author{
Benno M. Nigg ${ }^{1, *}$, Maurice Mohr' ${ }^{1}, \&$ Sandro R. Nigg ${ }^{1}$ \\ 1 Human Performance Laboratory, Faculty of Kinesiology, University of Calgary, Calgary, Canada \\ * Corresponding author: Benno M. Nigg, Human Performance Laboratory, Faculty of Kinesiology, The University of Calgary, \\ 2500 University Drive NW, Calgary (Alberta), T2N 1N4, Canada \\ Tel: +1 (403) 220 3436, Fax: +1 (403) 2827637 \\ E-Mail: nigg@ucalgary.ca
}

\section{TARGET ARTICLE}

\section{Article History:}

Submitted $13^{\text {th }}$ November 2018

Accepted $21^{\text {st }}$ November 2018

Published $7^{\text {th }}$ May 2019

Handling Editor:

Markus Tilp

University of Graz, Austria

Editor-in-Chief:

Martin Kopp

University of Innsbruck, Austria

\section{ABSTRACT}

Background: Six experts in the field of running-related research have critically addressed a proposal to abandon the paradigms of 'impact force' and 'pronation control' when investigating running shoes, running injury, and running performance. Further, these experts have commented on the suggestion of the new paradigms of 'muscle tuning' and the 'preferred movement path' that can be used to investigate questions related to running injuries and performance as well running shoe design and comfort. This publication synthesizes and addresses the main criticisms of the experts and describes future directions to further develop the 'muscle tuning and 'preferred movement' paradigms.

Keywords:

Impact forces - pronation - running biomechanics - running injuries - running performance running shoes

Citation:

Nigg, B. M., Mohr, M. \& Nigg, S. R. (2019). Response to select comments on the proposed paradigm shifts in running. Current Issues in Sport Science, 4:001. doi: 10.36950/CISS_2019.001.

Nigg, B. M., Mohr, M. \& Nigg, S. R. (2017). Muscle tuning and preferred movement path - a paradigm shift. Current Issues in Sport Science, 2:007. doi: 10.36950/CISS_2017.007. This is a response to commentaries on a CISS target article authored by Benno M. Nigg, Maurice Mohr, \& Sandro R. Nigg. For retrieving the whole target article including index of contents, editorial, main article, all peer commentaries and author's response: CISS_2019.100

\section{Introduction}

This publication is a reply to comments made by six experts in the field of running and running shoe construction (Becker, 2018; Clark, Udofa, Ryan, \& Weyand, 2018; Federolf, Doix, \& Jochum, 2018; Hamill, Boyer, \& Weir, 2018; Paquette \& Miller, 2018; Vanwanseele, Zhang, \& Schütte, 2018) regarding the proposal of replacing the paradigms of impact force and pronation control with some new paradigms (B.M. Nigg, Mohr, \& S.R. Nigg, 2017). The comments of the experts can be summarized as follows:

(1) External impact force variables have not been correctly assessed and/or their relationship with internal loading has not been considered. This has hampered the analysis of the association between impact forces and running injuries and thus, the 'impact force' paradigm should not be abandoned (Becker, 2018; Clark et al., 2018; Paquette \& Miller, 2018; Vanwanseele et al., 2018).

(2) The methods to quantify muscle tuning and the preferred movement path (PMP) along with the predictions that can be made from this paradigm need to be improved/ developed (Federolf et al., 2018; Hamill et al., 2018; Vanwanseele et al., 2018).

(3) The traditional variables to quantify pronation are not valid and do not adequately describe foot movement or the relative movement of foot and shank segments. Therefore, 
the topic of foot movement and injuries should be reevaluated (Becker, 2018; Vanwanseele et al., 2018).

(4) The association of the proposed new paradigms with running injuries was a main topic of all comments. In principal, we agree with comments 1-3 above. With respect to comment 4 , we agree that the proposed paradigms can be used to formulate hypotheses related to running injury mechanisms, but also want the readers to consider other potential outcomes like the effect on performance or comfort. We hope that the result of this process and these papers will lead to a better understanding of running related questions. Detailed replies to each of the four generalized comments follow.

\subsection{The difference between external and internal impact forces}

Running paradigms can be used to formulate hypotheses about mechanisms related to running injuries. Locomotion related injuries should always be discussed from the perspective of tissue loading and tissue adaptation (Hreljac, 2004; Paquette \& Miller, 2018). If the tissue is loaded in a way that results in a more rapid tissue breakdown compared to the rate of tissue regeneration, an injury of that tissue will follow eventually. If a runner increases his/her exercise volume and frequency too fast so that tissue adaptation and regeneration cannot occur, the runner will get injured. From this perspective, different mechanisms have been suggested for loading scenarios that will make an injury more likely to occur, e.g. high magnitude and rate of impact loading, the failure to adequately tune muscles, excessive foot movement, deviation from the preferred movement path and others. All these mechanisms are lacking experimental evidence regarding how they affect tissue-level strains. Therefore, we should acknowledge that efforts must be made to investigate the relationship between external variables (e.g. ground reaction forces, joint angles, segmental / soft-tissue accelerations) and tissue-level strains. This knowledge can be gathered by using 1) model calculations to determine internal forces and stresses in vivo (e.g. Wright, Neptune, van den Bogert, \& Nigg, 1998) and 2) mechanical tests to study the relationship between tissue loading profiles (i.e. magnitude, rate, frequency, duration) and tissue failure ex vivo (e.g. Edwards, 2018). One key result from the second approach illustrated that the influence of the ground reaction force loading rate on the fatigue behavior of bone may be negligible compared to the influence of the loading magnitude (Loundagin, Schmidt, \& Edwards, 2018). In other words, we now have evidence that increased loading rate may not correlate well to damage at the bone tissue level. By conducting similar investigations, we have the ability to systematically investigate hypotheses regarding the relationship between external loading rate signals and the risk for specific injuries.

Since the relationship between external ground reaction forces (which are integral variables) and internal forces (which are local variables) is typically not very strong, it seems inappropriate to use external force variables to predict the development of internal injuries. Most reviewers agreed on this, and thus statements like "excessive impact loading causes running injuries" should be avoided.

The original impact force paradigm has been formulated using the externally measured ground reaction forces and their maximum peak, the impact force peak, and/or their maximal or average first derivative, the (external) loading rate. It is well understood that the ground reaction force during landing in running is the sum of many different components, the two most important components being the acceleration of part of the lower leg and the accelerations of the rest of the human body. The contribution of the foot and leg has originally been described using the effective mass model (Denoth, 1986). The combination of these two major force components has been illustrated and explained and is well understood (Shorten \& Mientjes, 2011).There is no disagreementabout the combination of these forces into a resultant ground reaction force. The possible disagreement is the interpretation of the initial peak. Some researchers suggest that the impact force component and the force component due to the rest of the body should be considered separately (Shorten \& Mientjes, 2011; Clark et al., 2018). Two comments seem to be appropriate: (a) To determine the loading on an internal structure of the athlete's body, one needs a model that calculates/estimates these internal loadings and this model would, most likely, use the resultant ground reaction force as an input. (b) Experimenting with the effective mass model or with the actual lower leg initiated force component may be helpful in understanding how the impact force can be influenced and/or in understanding how certain shoe construction elements influence the external loading. However, to assess the actual loading of biological structures one needs a biomechanical model using the actual ground reaction forces as indicated above.

\subsection{The impact force paradigm should not be abandoned}

The impact force paradigm as currently formulated states that high external impact force peaks and high external loading rates are the reason for running injuries. We have shown that functional and statistical evidence for such a statement is missing. Consequently, we have argued that the impact paradigm in its current form, i.e. that the early peak in the external ground reaction force causes injuries per se should be abandoned.

We do not generally reject the idea that impact loading could be important for running mechanics and running injuries. Instead, we argue that the traditional measures of external impact loading are not useful in describing underlying biomechanical phenomena and thus should not be used as a starting point to investigate running mechanics and/or running injuries. The ground reaction force is a variable that has magnitude, frequency and variability. Theses aspects may and most likely will help us to understand the neuro-motor and loading aspects during running. Further, these aspects are not solely associated with running injuries, but rather relate in a much 
broader sense to the understanding of the biomechanical and neuro-motor aspects of running (Federolf et al., 2018). Too understand the risk factors for running injuries, however, we should concentrate on variables that quantify internal loading.

\subsection{Muscle tuning and predictions}

The fact that we adapt our muscles for any activity has not been challenged. However, the practical implication of the proposed muscle tuning paradigm remains unclear (Hamill et al., 2018; Vanwanseele et al., 2018). We are used to accept changes in muscle activity due to a specific change in movement (e.g. walking versus hopping). However, we are not used to think of such changes due to other reasons within the same movement task. Furthermore, the concept that vibrations of soft tissue compartments may affect our muscle activities is novel to many.

The authors agree that working on this topic exceeds the typical comfort zone of biomechanical studies. However, we think that this topic is important, especially from an energy and fatigue point of view. The athletes know what to expect when running and they adapt quickly to changing situations. We suggest that there are two reasons for changes in muscle activity; a) to satisfy the mechanical requirements when movement changes, and b) to minimize large soft tissue compartment vibrations. Our proposed "muscle tuning paradigm" suggests that runners activate specific muscles if the frequency of the input force (impact) is close to the natural frequency of these soft tissue compartments and the vibrations of these compartments could become excessive. "Reacting" corresponds to increasing the damping of these muscles or changing the natural frequency of the soft tissue compartment. Both situations correspond to a change in muscle activity and both situations correspond to a change of the muscle-tendon unit characteristics (Federolf et al., 2018). One major problem when working on the concept of muscle tuning is that these muscle activity changes cannot easily be differentiated. However, for running shoe construction one has theoretically two possibilities: (a) One can change the natural frequency of the soft tissue compartment by increasing/decreasing muscle activity and/or (b) one can shift the frequency of the input signal so that it is far away from the natural frequency by changing the running mechanics (e.g. the running kinematics or the running shoes). Based on results of initial experimental results, the first strategy of changing the natural frequency of the soft tissue compartments is rarely used by athletes. This leaves the second possibility, i.e. changing the input frequency. Preliminary experiments where shoe properties were altered with the objective of reducing soft-tissue vibrations, showed a decrease in vibration energy of $5 \mathrm{~dB}$, a substantial reduction when compared to normal running shoes. Two important additional comments in this context are 1) that the individually correct changes in input frequency can be an increase or a decrease in the input frequency depending on the runner's anthropometric characteristics, and 2) the strategies to move away from the resonance phenomena are individual by nature, meaning there is not one solution for all runners.

\subsection{Muscle tuning and running injuries}

It should be emphasized that the proposed muscle tuning paradigm is not equivalent to a possible explanation for an injury mechanism. Instead, the muscle tuning paradigm aims to describe basic neuromuscular strategies of the motor control system during movement. However, the paradigm can be used as a framework to formulate hypotheses about how the risk of injury can be reduced or how comfort or performance during running can be improved - particularly with a focus on how footwear can be used to achieve this. This means that questions in running biomechanics / motor control can be structured into one of two categories: (1) Questions that further advance our understanding of basic neuromuscular strategies during running or (2) questions about how certain biomechanical variables affect injury risk, performance and/or comfort. Based on our current knowledge and based on theoretical considerations we tend to see the application more in the energy/performance and comfort field than in the injury field.

\subsection{Preferred movement path and predictions}

The proposed paradigm of the "preferred movement path" was generally well accepted by the expert panel. The major concern was how to determine the preferred movement path (e.g. Hamill et al., 2018). Furthermore, it was suggested that the variability of the actual movement path should be included in further studies (Federolf et al., 2018; Vanwanseele et al., 2018). We agree that the concept of the preferred movement path is currently not easily quantified. At this point in time there is (in the view of the authors) no valid method available to determine and/or predict the preferred movement path. We suggest that the concept of the preferred movement should be investigated by analyzing not only the movement but include muscle activity in the experimental set-up. We recently showed that the muscle activation patterns of the vasti muscles during running may be highly variable but non-random between steps (von Tscharner, Ullrich, Mohr, Marquez, \& Nigg, 2018). This may reflect the strategy of the motor control system to maintain the preferred movement path by continuously adjusting the activity of leg muscles in response to slightly different external forces at each heel strike. The goal of further studies should be to investigate the link between variability in muscle activation patterns and variability in segment kinematics. According to the PMP, there should be a relationship between the two variables.

If the influence of external conditions on the runner is small, substantial neuromuscular adjustments to maintain the PMP are not required. In this case, the integrated muscle activity should be minimal. We suggest, that the measurement of running kinematics and muscle activation patterns in conjunction with global oxygen consumption could provide further insight 
into this prediction of the PMP and its relationship to energy expenditure.

Furthermore, it has been proposed (Federolf et al., 2018) that joint angles should not be viewed in isolation. Instead, the simultaneous coordination of multiple joints and segments should be of interest. Finally, it should be studied whether the preferred movement path changes or remains with increasing fatigue.

\subsection{Preferred movement path and running injuries}

The preferred movement path paradigm has been proposed to improve the understanding of the biomechanical and neurophysiological aspects of running (similar to the muscle tuning paradigm). Whether or not it is associated with the development of running injuries is not clear to the authors at this point in time. Theoretically, one should expect that running outside of one's preferred movement path would increase the muscular demand and, therefore, increase fatigue (Paquette \& Miller, 2018). Further research is needed to clarify this aspect.

\subsection{The traditional pronation variables do not adequately describe the foot movement}

There seems to be general agreement that the variables currently used to assess pronation are questionable. Further support for this general "feeling" has been provided through the results of a recent study analyzing 62 currently used "pronation variables" (Behling, von Tscharner, Manz, \& Nigg, 2018). The results of this study showed no strong or moderate correlation between different variables. This means that the commonly used variables used to describe the term pronation, are actually describing different aspects of foot movement and may even not be associated with foot pronation. There seems to be agreement in the responses of the experts that this aspect requires further analysis and that the current approaches are not helpful in understanding "pronation" and its relationship (if any) with running injuries. We suggest that further studies should concentrate on "foot movement" and not on "pronation", and that the foot movement should be segmented into different aspects (forefoot, midfoot, and calcaneal), in order to better understand each segment of the foot, and the relationship between those segments.

\subsection{Pronation and running injuries}

The epidemiological studies assessing "pronation" as a risk for running injuries show the same problems as the epidemiological studies for impact loading. The studies use typically small subject samples and consequently, the results are random and can not be used for strong predictions. There is, however, one study that has a large sample (927 participants and 1854 feet) that provides enough statistical power to answer the injury risk question (Nielsen et al., 2014). They showed that people that "pronate" have the lowest injury risk. Furthermore, they showed that people that have excessive pronation only represent about $1 \%$ of the population, and these individuals did trend to being susceptible to injury. The results of this study seem to answer the question whether "pronation" is an injury risk - yes if extreme, no for the average runner. However, the study has some short comings. The "pronation" was quantified using the FPI (foot posture index). The FPI is a static variable and is one of the variables that does not have any correlation with the other commonly used "pronation" variables. Thus, we don't know, whether or not the FPI actually quantifies "pronation". Consequently, it is fair to state that any proposed injury risk factors related to "pronation" should be treated with caution, unless we are dealing with a small sub segment of the population.

We suggest that a good understanding of the detailed foot movement (forefoot, midfoot and rearfoot) and its relationship to internal tissue loading (Becker, 2018) may help to improve the understanding of possible injury mechanisms.

\section{The proposed paradigms and running injuries}

In our opinion (and in agreement with Becker, 2018 and Paquette \& Miller, 2018) injuries should always be discussed from the perspective of tissue loading and tissue adaptation. Efforts must be made to investigate tissue-level strains or local loading.

It is important, however, to avoid generalizing statements about running injuries such as'excessive foot movement causes injuries' or "gait training reduces injuries in novice runners" (Chan et al., 2018). Hypotheses must be specific for individual running-related injuries. For example:'Tibialis posterior muscle weakness may result in lower longitudinal arch stiffness and therefore higher plantar fascia strain during running and thus plantar fasciitis'. Or: 'The necessity to tune the calf muscles, e.g. when the input force signal during running would otherwise cause resonance effects, increases calf muscle strain and subsequently causes calf strain injuries'. Such hypotheses would be helpful to advance our understanding, the prevention and treatment of running injuries.

In summary, more studies for improving the understanding of running injuries are needed. Large prospective epidemiological studies using model calculations to determine internal loading may not be feasible. Therefore, one may want to structure future running injury research in two steps: (a) Studies with relatively small sample sizes under controlled conditions to identify external biomechanical variables that are highly correlated with internal loading and (b) Large epidemiological studies that use these external variables, restricted to one injury based on a functional understanding of the possible injury mechanism. 


\section{Funding}

The author has no funding or support to report.

\section{Competing Interests}

The authors have worked over about 40 years with more than 40 industry partners. Many of these contacts were paid for by these partners. Results and understanding from projects with these partners have influenced the development of the paradigms, presented in this paper. However, the authors declare that no competing interests exist.

\section{Data Availability Statement}

All relevant data are within the paper.

\section{References}

Becker, J. (2018). New Hypotheses and Unanswered Questions in Running Injury Research - comment on Nigg et al. Current Issues in Sport Science (CISS), 3:102. doi: 10.15203/ CISS_2018.102

Behling, A.-V., von Tscharner, V., Manz, S., \& Nigg, B. M. (2018). Longitudinal Arch Angle and Achilles Tendon Angle dominate Foot Movement. Presented at the Annual Alberta Biomedical Engineering Conference, Banff, Alberta.

Chan, Z. Y. S., Zhang, J. H., Au, I. P. H., An, W. W., Shum, G. L. K., Ng, G. Y. F., \& Cheung, R. T. H. (2018). Gait Retraining for the Reduction of Injury Occurrence in Novice Distance Runners: 1-Year Follow-up of a Randomized Controlled Trial. The American Journal of Sports Medicine, 46(2), 388-395. doi: 10.1177/0363546517736277

Clark, K. P., Udofa, A. B., Ryan, L. J., \&Weyand, P. G. (2018). Running impact forces: from half a leg to holistic understanding comment on Nigg et al. Current Issues in Sport Science (CISS), 3:107. doi: 10.15203/CISS_2018.107

Denoth, J. (1986). Load on the locomotor system and modeling. In Biomechanics of Running Shoes (pp. 63-116). Champaign, IL: Human Kinetics Publishers.

Edwards, W. B. (2018). Modeling Overuse Injuries in Sport as a Mechanical Fatigue Phenomenon. Exercise and Sport Sciences Reviews, Publish Ahead of Print. doi: 10.1249/ JES.0000000000000163

Federolf, P., Doix, A.-C. M., \& Jochum, D. (2018). A discussion of the Muscle Tuning and the Preferred Movement Path concepts - comment on Nigg et al. Current Issues in Sport Science (CISS), 3:103(0). doi: 10.15203/CISS_2018.103

Hamill, J., Boyer, K. A., \& Weir, G. (2018). A paradigm shift is necessary to relate running injury risk and footwear design - comment on Nigg et al. Current Issues in Sport Science (CISS), 3:104. doi: 10.15203/CISS_2018.104
Hreljac, A. (2004). Impact and overuse injuries in runners. Medicine and Science in Sports and Exercise, 36(5), 845-849.

Loundagin, L. L., Schmidt, T. A., \& Edwards, W. B. (2018). Mechanical Fatigue of Bovine Cortical Bone Using Ground Reaction Force Waveforms in Running. Journal of Biomechanical Engineering, 140(3), 031003-031003-031005. doi: 10.1115/1.4038288

Nielsen, R. O., Buist, I., Parner, E. T., Nohr, E. A., Sørensen, H., Lind, M., \& Rasmussen, S. (2014). Foot pronation is not associated with increased injury risk in novice runners wearing a neutral shoe: a 1-year prospective cohort study. Br J Sports Med, 48(6), 440-447. doi: 10.1136/bjsports-2013-092202

Nigg, B. M., Mohr, M., \& Nigg, S. R. (2017). Muscle tuning and preferred movement path - a paradigm shift. Current Issues in Sport Science (CISS), 2:007. doi: 10.36950/CISS_2017.007

Paquette, M. R., \& Miller, R. H. (2018). Reconciling new with old injury paradigms and the need to dig deeper - comment on Nigg et al. Current Issues in Sport Science (CISS), 3:105. doi: 10.15203/CISS_2018.105

Shorten, M., \& Mientjes, M. I. V. (2011). The 'heel impact' force peak during running is neither 'heel' nor 'impact' and does not quantify shoe cushioning effects. Footwear Science, 3(1), 41-58. doi: 10.1080/19424280.2010.542186

Vanwanseele, B., Zhang, X., \& Schütte, K. (2018). Muscle tuning and preferred movement path: do we need a paradigm shift or should we redefine the old? - comment on Nigg et al. Current Issues in Sport Science (CISS), 3:106. doi: 10.15203/ CISS_2018.106

von Tscharner, V., Ullrich, M., Mohr, M., Marquez, D. C., \& Nigg, B. M. (2018). A wavelet based time frequency analysis of electromyograms to group steps of runners into clusters that contain similar muscle activation patterns. PLOS ONE, 13(4), e0195125. doi: 10.1371/journal.pone.0195125

Wright, I. C., Neptune, R. R., van den Bogert, A. J., \& Nigg, B. M. (1998). Passive regulation of impact forces in heel-toe running. Clinical Biomechanics, 13(7), 521-531. doi: 10.1016/ S0268-0033(98)00025-4 